\title{
Sacrificial Tamper Slows Down Sample Explosion in FLASH Diffraction Experiments
}

\author{
Stefan P. Hau-Riege, ${ }^{1, *}$ Sébastien Boutet, ${ }^{2}$ Anton Barty, ${ }^{1,6}$ Saša Bajt, ${ }^{3}$ Michael J. Bogan, ${ }^{2}$ Matthias Frank, ${ }^{1}$ \\ Jakob Andreasson, ${ }_{4}^{4}$ Bianca Iwan, ${ }^{4}$ M. Marvin Seibert, ${ }^{4}$ Janos Hajdu, ${ }^{4}$ Anne Sakdinawat, ${ }^{5}$ Joachim Schulz, ${ }^{6}$ \\ Rolf Treusch, ${ }^{3}$ and Henry N. Chapman ${ }^{6,7}$ \\ ${ }^{1}$ Lawrence Livermore National Laboratory, 7000 East Avenue, Livermore, California, 94550, USA \\ ${ }^{2}$ SLAC National Accelerator Laboratory, 2575 Sand Hill Road, Menlo Park, California 94025, USA \\ ${ }^{3}$ HASYLAB, Deutsches Elektronen-Synchrotron DESY, Notkestraße 85, 22607 Hamburg, Germany \\ ${ }^{4}$ Laboratory of Molecular Biophysics, Department of Cell and Molecular Biology, Uppsala University, \\ Husargatan 3, Box 596, SE-75124 Uppsala, Sweden \\ ${ }^{5}$ University of California, Berkeley, 253 Cory Hall, Berkeley, California 94720, USA \\ ${ }^{6}$ Center for Free Electron Laser Science, DESY, Notkestraße 85, 22607 Hamburg, Germany \\ ${ }^{7}$ Universität Hamburg, Luruper Chaussee 149, 22761 Hamburg, Germany
}

(Received 16 November 2009; published 12 February 2010)

\begin{abstract}
Intense and ultrashort x-ray pulses from free-electron lasers open up the possibility for near-atomic resolution imaging without the need for crystallization. Such experiments require high photon fluences and pulses shorter than the time to destroy the sample. We describe results with a new femtosecond pumpprobe diffraction technique employing coherent $0.1 \mathrm{keV}$ x rays from the FLASH soft $\mathrm{x}$-ray free-electron laser. We show that the lifetime of a nanostructured sample can be extended to several picoseconds by a tamper layer to dampen and quench the sample explosion, making $<1 \mathrm{~nm}$ resolution imaging feasible.
\end{abstract}

PACS numbers: $41.60 . \mathrm{Cr}$

Single-particle coherent diffractive $\mathrm{x}$-ray imaging aims to achieve near-atomic resolution for atomic clusters, macromolecules, macromolecular complexes, or larger objects without the need for crystallization. Despite the short pulse lengths offered by $\mathrm{x}$-ray free-electron laser (XFEL) sources, the achievable resolution of images will depend on the degree of radiation damage to the molecule that occurs during the course of the $\mathrm{x}$-ray pulse. High $\mathrm{x}$-ray fluences ( $\sim 10^{12}$ photons/molecule) are necessary to compensate for the small scattering strength of single molecules [1]; meanwhile high fluences also result in more rapid x-ray damage to the molecule. We propose to retard this damage, and thereby relax the pulse length requirement, by encapsulating the molecule in a thin sacrificial tamper layer $[2,3]$. The tamper layer supplies a bath of photoinduced free electrons to the sample, and arrests the hydrodynamic expansion through inertial confinement.

The calculated explosion of a homogeneous particle of $150 \AA$ diameter irradiated by a 25 fs-long XFEL pulse of $8 \mathrm{keV}$ x rays (similar to those generated at Stanford's Linac Coherent Light Source [4]) is shown in Fig. 1. The calculations were performed using the hydrodynamic model described in Ref. [5]. We chose the pulse fluences such that the total intensity scattered by the sample is the same for this case and our experimental investigation with $0.1 \mathrm{keV}$ FEL pulses obtained at FLASH [6] at a wavelength of $13.5 \mathrm{~nm}$ and a fluence of $31 \mathrm{~J} / \mathrm{cm}^{2}$. Note, in particular, how the untampered particle shape, Fig. 1(a), significantly changes within a few femtoseconds according to the simulations. A sacrificial tamper layer helps retard this damage. In a likely scenario for single-particle imaging, multiple tamper-coated molecules are injected into the XFEL beam one by one, and the measured diffraction patterns averaged to improve the signal-to-noise ratio [7-10]. Our hydrodynamic simulations of the present FLASH experiment suggest that without a tamper, the sample disintegrates on the time scale of a few picoseconds whereas with a tamper the sample more-or-less retains its shape, as shown for the case of soft XFEL pulses in Figs. 1(b) and 1(c). The simulation of the temperature and density evolution of the samples irradiated with $0.1 \mathrm{keV}$ x-ray pulses was calculated using the HYDRA radiation hydrodynamics code that accounts for radiation transport and electron thermal conduction [11]. We based our estimate of the complex index of refraction on published room-temperature solid-density values and corrected for changes with temperature and density using an average ion model employing screened hydrogen potentials [12]. Tampering could lead to atomic resolution structure determination in "diffraction before destruction" experiments at short wavelengths. This will enable groundbreaking new experiments in biology, cluster physics, nanoscale chemistry, or in studies on matter under extreme conditions.

Here, we present direct experimental evidence that a sacrificial tamper layer can be successful in containing hydrodynamic expansion during the XFEL pulse using a variation of the femtosecond time-delay holography technique [13]. This technique exploits the fact that a multilayer mirror can reflect back a FEL pulse at $13.5 \mathrm{~nm}$ wavelength before the mirror surface is destroyed by that extremely intense FEL pulse [14]. By reflecting the FEL beam back onto itself, the same beam can be used both to trigger the sample explosion and then to probe the exploding sample with a well-defined time delay. An innovation 
(a) $150 \AA$ aluminum sphere, $8 \mathrm{keV}$ FEL pulse:

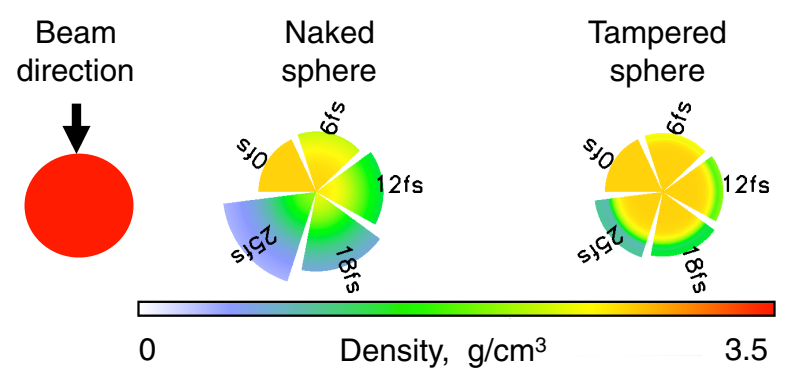

(b) Naked aluminum pillar, $0.1 \mathrm{keV}$ FEL pulse:

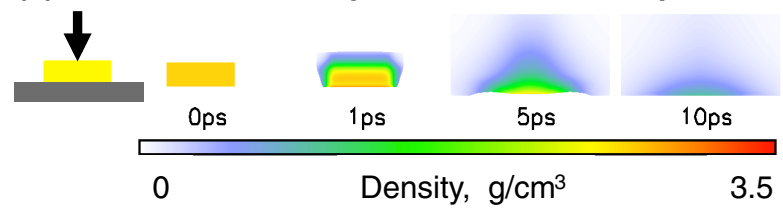

(c) Tampered aluminum pillar, $0.1 \mathrm{keV}$ FEL pulse:

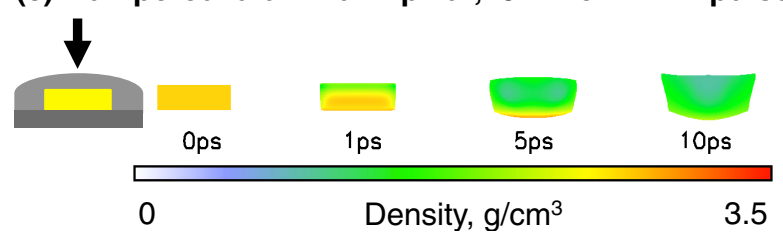

FIG. 1 (color online). Calculation of the expansion dynamics. (a) A $150 \AA$-diameter aluminum sphere during XFEL irradiation with a fluence of $2.5 \times 10^{8} \mathrm{~J} / \mathrm{cm}^{2}$ without a tamper (left) and with a $25 \AA$ thick silicon tamper (right). (b),(c) Cross-sectional view of a $70 \mathrm{~nm}$-tall and $200 \mathrm{~nm}$-wide aluminum cylinder after XFEL radiation with a fluence of $30 \mathrm{~J} / \mathrm{cm}^{2}$ (b) without a tamper and (c) with a $75 \mathrm{~nm}$ thick silicon tamper. Only the aluminum portion of the sample is shown. We chose the pulse fluences such that the total intensity scattered by the sample is the same for the 8 and $0.1 \mathrm{keV}$ case. The pulse duration was $25 \mathrm{fs}$.

used here (Fig. 2) is that we placed samples on very small sample windows, which prevent the primary diffracted light from reaching the detector after it is reflected back from the mirror. Figure 2 shows the experimental arrangement. The small window apertures the reflected beam, thereby removing the scattering from the pump and allowing only the scattering from the probe to reach the detector. The back-reflecting mirror was mounted on a five-axis stage which allowed precise control of the sample-tomirror distance and therefore the time delay. The mirror mount was equipped with fine pitch and yaw control which allowed the back-reflected beam to be steered through the very small opening $(7.5 \mu \mathrm{m})$ of the sample window with the mirror located many millimeters away, ensuring the spatial overlap of the two passes of the beam at the sample plane. This overlap was verified using beam-based alignment with the attenuated beam. The technique requires a detector system that has a hole in the middle to let the incident beam access the sample while covering the desired solid angle. The detector system used was described in detail in Ref. [15]. For very small distances $\Delta z$, the solid angle from the mirror through the sample window is larger, and the diffracted light from the first and second irradiation of the sample coherently combine giving rise to "dusty mirror" interference rings [13]. These circular fringes were observed for very short time delays here, when the mirror and the sample were brought in contact with each other. The radii of the circular fringes were used to precisely calibrate the time delay with a few fs accuracy. For larger distances $\Delta z$, only the diffracted light from the second irradiation is recorded since the back-reflected diffracted beam is collimated within the central hole in the detector. The time delay for larger sample-to-mirror distances, where the Newton rings were not visible, was calculated with high precision by simply knowing the mirror position relative to the short time-delay position. Using small window sizes has the further advantages that the data analysis is significantly simplified and that the overlap of the pump and probe beam is ensured. The FEL-pump and FEL-probe technique used here is currently the only method that enables imaging of the sample during the course of the explosion at a precise time point after $\mathrm{x}$-ray excitation.

Hydrodynamic simulations indicate that the physics describing the macroscopic motion of spherical and planar samples is equivalent. Samples for measuring the hydrodynamic explosion rate used here consisted of cylindrical aluminum pillars deposited on $100 \mathrm{~nm}$-thick silicon-nitride membranes, spanning $7.5 \mu \mathrm{m}$ square windows etched into a silicon wafer [see inset in Fig. 3(c)]. The membrane windows were fabricated by lithographically defining square-shaped holes on the backside of a $\mathrm{Si}_{3} \mathrm{~N}_{4}$-coated wafer, and removing the silicon anisotropically in a potassium hydroxide $(\mathrm{KOH})$ etch bath. The aluminum pillars were fabricated using a lift-off process on electron-beamevaporated aluminum. The diameter of the aluminum pillars was $200 \mathrm{~nm}$ and the height was $70 \mathrm{~nm}$. Ten identical aluminum pillars were positioned on each window in a

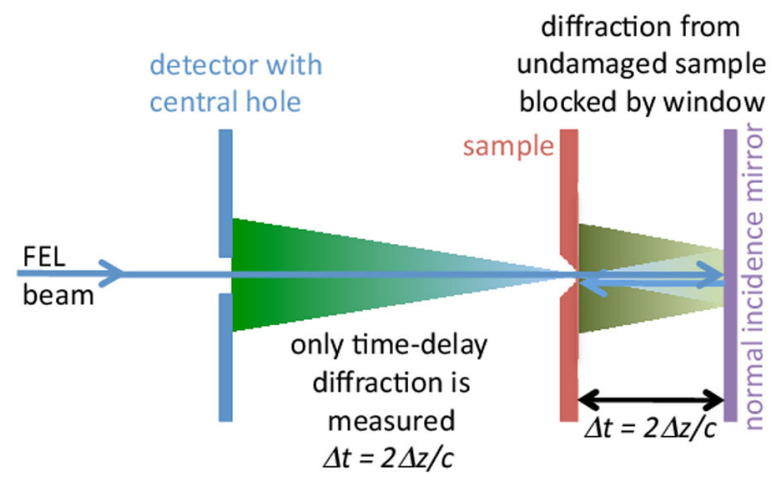

FIG. 2 (color online). Scattering geometry of the one-color pump-probe experiments. The FEL beam irradiates the sample, initiating the reaction, and reflects from a normal-incidence multilayer mirror back onto the sample [9]. The FEL beam once again illuminates the sample with a time delay of $\Delta t=$ $2 \Delta z / c$, probing the reaction. The prompt diffraction signal is blocked using a small sample window. In this way only diffracted light from the time-delayed sample is detected using a charge-coupled device (CCD) on the left. 
predefined pattern within a $1.5 \mu \mathrm{m}$ extent. This object size was smaller than the determined $12-\mu \mathrm{m}$ full-width-at-halfmaximum (FWHM) FEL beam diameter in the focus of beam line BL2 at FLASH [16] to ensure uniform intensity across the sample. Some of the membranes were coated with a $100 \mathrm{~nm}$-thick silicon tamper layer which is optically transparent at the probe wavelength $(13.5 \mathrm{~nm})$. This configuration was selected to closely mimic the case of a hardXFEL imaging experiment of molecules embedded in a tamper film, but on length scales that are accessible at $13.5 \mathrm{~nm}$ wavelength at FLASH.

Far-field diffraction patterns measured with and without a tamper for time delays of $25 \mathrm{fs}$ and $13.5 \mathrm{ps}$ are shown in Figs. 3(a) and 3(b). A circular pillar gives rise to a diffrac-

(a) Explosion of naked sample

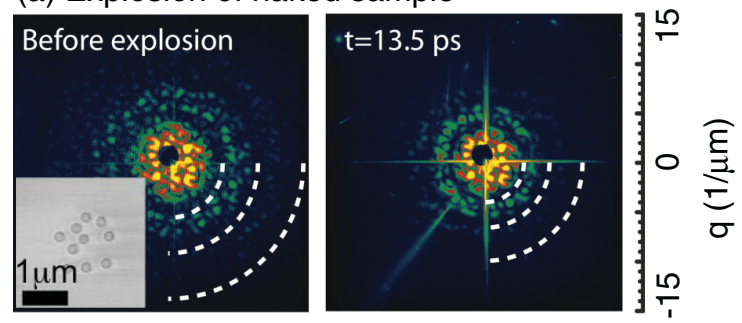

(b) Retarded explosion of tampered sample
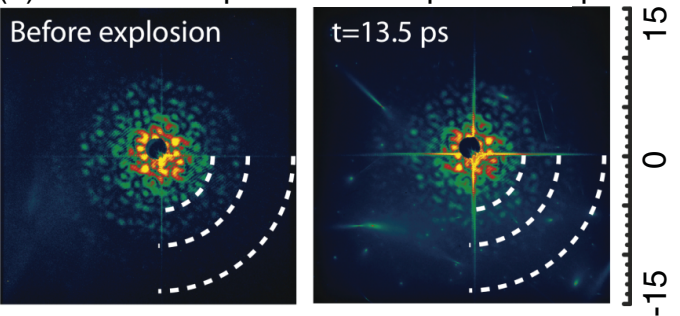

$\underbrace{\stackrel{E}{E}}_{\sigma}$

(c) Difference between unexploded and $\mathrm{t}=13.5 \mathrm{ps}$ images

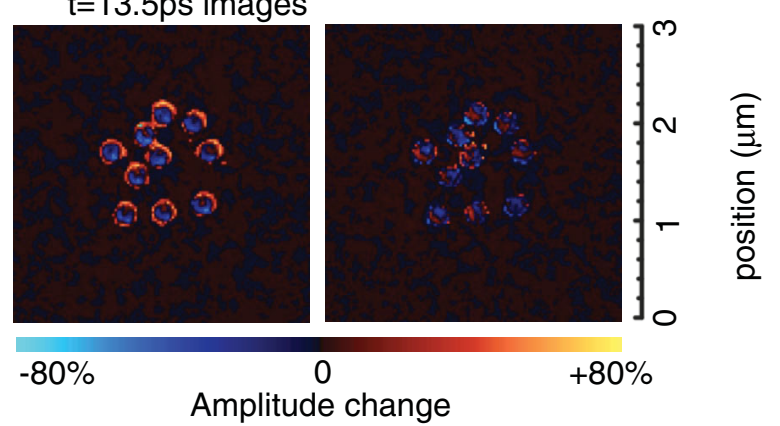

FIG. 3 (color). Measured far-field diffraction patterns and reconstructed structures. (a) Diffraction from the naked, untampered sample (with an SEM picture on the left of the sample before exposure), and (b) from the tampered sample. Arcs highlight Airy-like diffraction minima in the measured data. Contraction of the arcs indicates sample expansion. This is significant in (a) and small in (b). (c) Difference images (13.5 ps vs $25 \mathrm{fs)}$ of reconstructed structures. Light colors indicate significant density changes. The untampered sample (left) shows large expansion, whereas the tampered sample (right) shows minimal structural changes. The wavelength was $13.5 \mathrm{~nm}$, the pulse length $25 \mathrm{fs}$, and the fluence $31 \mathrm{~J} / \mathrm{cm}^{2}$. tion pattern similar to Airy's rings with the angular positions of intensity minima inversely proportional to the pillar radius. In our experiment this single-pillar pattern is modulated by speckles that encode the arrangement of the identical pillars. The expansion of the pillars causes a change in the Airy-like envelope of the diffraction pattern. The diffraction patterns before the explosion are averages over multiple low-fluence exposures during which the sample did not change significantly (the "unperturbed" sample). We use these patterns as a basis for comparison. The diffraction patterns with 13.5 ps time delay were obtained from single-pulse high-fluence exposures. The difference between tampered and untampered sample is immediately apparent. With a tamper layer present, the diffraction patterns of the low- and high-fluence exposures are similar and the diffraction Airy-like minima are at similar positions. Without a tamper layer, the diffraction minima have shifted to significantly smaller angles, indicating that the aluminum pillars have expanded. Reconstruction of the diffraction patterns using iterative phase retrieval enables us to directly image the particle expansion. Image reconstruction was carried out using iterative transform phase retrieval techniques. We used the RAAR algorithm $[17,18]$ coupled with the SHRINKWRAP dynamic support refinement algorithm [19]. The object is allowed to be complex valued to take into account phase aberrations in the illuminating beam and phase structure in the object. Initial support was based on thresholding the object autocorrelation, computed by the Fourier transform of the measured diffraction pattern at a time delay of $\Delta t=0$, and then allowed to evolve dynamically. The support constraint is calculated every 100 iterations by selecting pixels with intensity values greater than $10 \%$ of the maximum image intensity, after first blurring the image with a Gaussian kernel. The algorithm was halted after 3000 iterations. A tight support was generated from the solution which was obtained this way. The final solution is the average of 100 separate solutions obtained from 100 random phase starts using the tight support described above. To assist in image reconstruction the solution and support obtained for $\Delta t=0$ was used as an initial guess for phase retrieval at longer time delays, and then allowed to evolve into the solution for the delayed $\Delta t>0$ diffraction pattern using the same algorithm and procedures described above.

Figure 3(c) shows the difference of reconstructed images before and during the explosion, both with a tamper around the aluminum pillars and without, giving even more detailed information about the expansion process.

Analysis of the diffraction pattern minima with and without a silicon tamper layer reveals further information about the hydrodynamic explosion. Figure 4 shows the resolution length $d$ of the diffraction pattern minima as a function of delay time for both cases. The resolution length is related to the scattering vector length $q$ by $d=2 \pi / q$, with $q=4 \pi \sin (\theta / 2) / \lambda$, where $\lambda$ is the wavelength and $\theta$ the scattering angle. Without a silicon tamper layer, $d$ drastically increases with increasing delay $\Delta t=2 \Delta z / c$, 


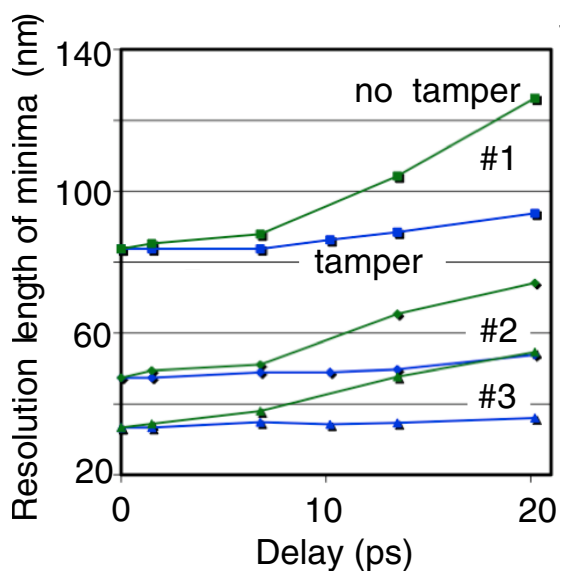

FIG. 4 (color online). Positions of diffraction minima. Resolution length of the first three Airy-like diffraction minima (labeled \#1, \#2, and \#3) of the aluminum pillars as a function of delay and with and without tamper.

indicating that the pillar expands. With a silicon tamper layer, the increase in $d$ is much more modest. This experiment demonstrates that a tamper layer ensures the integrity of the sample for a period of at least 5 ps duration following irradiation with a 25 fs-long $13.5 \mathrm{~nm}$ FEL pulse, which is $200 \times$ longer than the pulse duration.

These experimental results give a basis for estimating the effect of a sacrificial tamper layer in biomolecular imaging. When a hard x-ray FEL with a photon energy of $8 \mathrm{keV}$ irradiates a biological nanoparticle with a fluence of $10^{12}$ photons per pulse focused to a $100 \mathrm{~nm}$ spot, each nonhydrogen atom of the sample will absorb about one $\mathrm{x}$-ray photon on average. The highly energetic photoelectrons will escape from small samples, so that each absorbed x-ray photon contributes one Auger electron of energy $\sim 260 \mathrm{eV}$ to heating the particle. In comparison, the experiment at FLASH deposited $\sim 450 \mathrm{eV} /$ atom in the aluminum pillars (as calculated for a beam diameter: $\sim 12 \mu \mathrm{m}$ FWHM, average pulse energy: $24 \pm 6.6 \mu \mathrm{J}$, and cold opacities). The results show the tamper layer effectively prevented hydrodynamic expansion of the aluminum pillars. After $10 \mathrm{ps}$, the expansion of the aluminum pillars was less than $10 \mathrm{~nm}$, so that simple linear scaling to a pulse length of $25 \mathrm{fs}$ may suggest that a tamper restrains motion to about $1 \AA$ during a 25 fs pulse. Extrapolating to the biological case above, a tamper may efficiently delay hydrodynamic expansion of a $50 \AA$ diameter macromolecule on a time scale of $25 \mathrm{fs}$, which is a typical pulse length expected on upcoming hard x-ray FELs.

A likely tamper candidate for biomolecules is water. It has been used successfully in single-particle electron cryomicroscopy in the form of vitreous ice surrounding macromolecules, viruses and other objects. In XFEL experiments macromolecules could either be placed in a sheet of ice, corresponding to the tamper sheet geometry demonstrated here, or injected into vacuum in liquid drops [20] which create a tamper layer around the molecule. An alternative to water is the use of graphene sheets [21], which can act as electron suppliers to quench the explosion and reduce damage. These approaches are based on similar principles, i.e., supplying electrons to neutralize an ionized core combined with various degrees of inertial confinement, and offer a means to delay the sample explosion.

We would like to thank the scientific and technical staff at FLASH at DESY. Also thanks to C. Bostedt, T. Möller, H. Thomas, D. Rupp, S. Schorb, M. Adolph, all of T. U. Berlin, for discussions, technical assistance, and providing instrumentation. This work performed under the auspices of the U.S. Department of Energy by Lawrence Livermore National Laboratory under Contract No. DE-AC5207NA27344, the SLAC National Accelerator Laboratory under Contract No. DE-AC02-76SF00515, and the Deutsches Elektronen-Synchrotron, a research center of the Helmholtz Association. Additional support comes from the DFG Cluster of Excellence at the Munich Centre for Advanced Photonics [22], from the Virtual Institute Program of the Helmholtz Society, the Joachim Herz Stiftung, and from the Swedish Research Council.

*Corresponding author. hauriege1@1lnl.gov

[1] R. Neutze, W. Wouts, D. van der Spoel, E. Weckert, and J. Hadju, Nature (London) 406, 752 (2000).

[2] S. P. Hau-Riege, R. A. London, H. N. Chapman, A. Szoke, and N. Timneanu, Phys. Rev. Lett. 98, 198302 (2007).

[3] A. Mikaberidze, U. Saalmann, and J. M. Rost, Phys. Rev. A 77, 041201(R) (2008).

[4] Y. Ding et al., Phys. Rev. Lett. 102, 254801 (2009).

[5] S. P. Hau-Riege, R. A. London, and A. Szoke, Phys. Rev. E 69, 051906 (2004).

[6] W. Ackermann et al., Nat. Photon. 1, 336 (2007).

[7] G. Huldt, A. Szoke, and J. Hajdu, J. Struct. Biol. 144, 219 (2003).

[8] G. Bortel and G. Faigel, J. Struct. Biol. 158, 10 (2007).

[9] R. Fung, V. Shneerson, D. Saldin, and A. Ourmazd, Nature Phys. 5, 64 (2009).

[10] V. Elser, arXiv:0709.3858v1.

[11] M. M. Marinak et al., Phys. Plasmas 8, 2275 (2001).

[12] S. P. Hau-Riege, R. A. London, H. N. Chapman, and M. Bergh, Phys. Rev. E 76, 046403 (2007).

[13] H. N. Chapman et al., Nature (London) 448, 676 (2007).

[14] S. P. Hau-Riege et al., Phys. Rev. Lett. 98, 145502 (2007).

[15] S. Bajt et al., Appl. Opt. 47, 1673 (2008).

[16] S. Hau-Riege et al., Appl. Phys. Lett. 95, 111104 (2009).

[17] D. R. Luke, H.H. Bauschke, and P.L. Combettes, in Proceedings of the IEEE 2005 International Conference on Acoustics, Speech and Signal Processing, Philadelphia, PA, 2005 (IEEE, New York, 2005).

[18] H. N. Chapman et al., J. Opt. Soc. Am. A 23, 1179 (2006).

[19] S. Marchesini et al., Phys. Rev. B 68, 140101(R) (2003).

[20] D. P. DePonte et al., J. Phys. D 41, 195505 (2008).

[21] E. E. Fill, F. Krausz, and M. G. Raizen, New J. Phys. 10, 093015 (2008).

[22] www.munich-photonics.de 